Proceedings of the 2011 Winter Simulation Conference

S. Jain, R.R. Creasey, J. Himmelspach, K.P. White, and M. Fu, eds.

\title{
A MULTI-METHODOLOGY AGENT-BASED APPROACH FOR CONTAINER LOADING
}

\author{
Navonil Mustafee \\ Eberhard E. Bischoff \\ School of Business and Economics \\ Swansea University \\ Swansea, Wales, SA2 8PP, UK
}

\begin{abstract}
The paper deals with container loading and contends that combining Container Loading Algorithms (CLAs) with Agent-Based Simulation (ABS) is a feasible and useful way of analyzing trade-offs between loading efficiency and various practical considerations in relation to the cargo - such as its stability, fragility, or possible cross-contamination between items over time. The latter perspective is used to demonstrate the merits of the approach. More specifically, the paper considers a situation where the items involved have differing degrees of perishability and badly deteriorated items can affect others (e.g., through mold spreading). The output of the CLAs is used to create agents that simulate the spread of mold through proximity-based interactions. The results show the trade-offs between container utilization and the propagation of mold and demonstrate that there is not necessarily any correlation between these two factors. The key contribution of the research is the multi-methodology agent-based approach to container loading.
\end{abstract}

\section{INTRODUCTION}

Operations Research (OR) is the application of analytical methods that enable stakeholders to take informed decisions. Commonly used OR techniques include data envelopment analysis, cutting/packing optimization, vehicle routing, integer programming, statistical analysis, meta-heuristics, simulation and soft OR (Beasley 1996). In this paper we bring together two such techniques - Cutting and Packing Optimization (CPO) and Simulation - and study this in the context of container loading. More specifically, we focus on a particular category of CPO algorithms, namely, Container Loading Algorithms (CLAs), and a specific simulation technique, namely, Agent-Based Simulation (ABS), and propose a multi-methodology agent-based approach for container loading.

The broad heading of Container Loading covers a wide spectrum of scenarios which arise in practice. The cargo to be loaded may consist, for example, of identical items (completely homogenous cargo: Han, Knott, and Egbelu 1989; George 1992), a large number of different types of items relative to the total number of items (strongly heterogeneous cargo: Gehring, Menschner, and Meyer 1990), or relatively few different types of items (weakly heterogeneous cargo: Morabito and Arenales 1994; Ngoi, Tay, and Chua 1994; Wang, Li and Levy 2008). The problem may involve loading a consignment of goods into either a single or multiple containers; the items might all be rectangular in shape, cylindrical, or completely irregular; there may be different drop-off points for certain parts of the cargo etc. (Bischoff and Ratliff 1995). Common to all these types of situation is the overall objective of determining loading arrangements which are cost-effective according to some measure that is appropriate in the circumstances. In a basic scenario this might mean generating loading plans that allow for the maximum utilization of the container space. The term CLA is used here to describe any approach which is designed to produce container loading plans. Numerous different methods have been put forward in the literature (Pisinger 2002). 


\section{Mustafee and Bischoff}

In this paper we extend existing research in container loading by proposing a multi-methodology approach that combines CLAs and ABS. The approach enables us to analyze the trade-offs between loading efficiency and various cargo-related considerations. While loading efficiency is determined by the container loading plans generated by the CLAs, we propose the use of ABS to simulate the latter. For example, the stability of cargo may be determined by simulating the proximity-based interactions continually taking place among the individual items of cargo as they are being transported. Another example is the simulation of possible cross-contamination between different items of cargo over time. We use these two example scenarios to conduct a feasibility study of our multi-methodology approach to container loading.

The remainder of the paper is structured as follows. Section 2 presents a brief overview of CLAs. This is followed by a description of ABS simulation and a review of the associated literature (Section 3). Our multi-methodology agent-based approach for container loading is discussed in Section 4. Section 5 is devoted to the agent-based simulation model that we developed as part of our feasibility study; Section 6 discusses the experiments and presents the results. Section 7 is the concluding section of this paper.

\section{OVERVIEW OF CONTAINER LOADING ALGORITHMS}

The efficient loading of cargo into freight containers - and, more generally, the proficient packing of smaller items into larger objects - has been a subject of intensive research for at least thirty years. George and Robinson (1980) were among the first to propose an algorithm for constructing a container loading plan. Their approach was heuristic in nature and based on the idea of building a series of 'walls' of items across the width and height of the container. Since then numerous different approaches have been developed for both the knapsack version of the problem - where the space available is fixed and loading all the cargo may not be possible - and, to a lesser extent, its bin-packing form - where all of the cargo involved must be stowed and a cost-effective way of using a set of containers is sought. The work has made use of a wide variety of techniques, ranging from single-pass heuristics to genetic algorithms, tabu search, linear programming, random search and combinations of tree-search heuristics and dynamic programming. Parreño et al. (2008), Wang, Li and Levy (2008), Huang and He (2009) as well as Fanslau and Bortfeldt (2010) and Egeblad et al. (2010) present useful, recent overviews of the research. A more comprehensive literature review is provided by Pisinger (2002), and Wäscher, Haußner, and Schumann (2007) discuss the position of this work in relation to the wider literature on CPO.

Much of the more recent work has moved away from pure knapsack or bin-packing formulations of the container loading problem and has paid increasing attention to various additional factors which may affect the task in practice. Orientation constraints on individual types of cargo (Fanslau and Bortfeldt 2010) and container weight capacity limits (Gehring and Bortfeldt 2002) represent simple examples of such factors. The literature has also used problem definitions which include the weight distribution within a container as a critical factor (Gehring and Bortfeldt 1997, Davies and Bischoff 1999, Eley 2002) and aspects of cargo stability have been explicitly considered in several approaches as attributes of solution quality (Bortfeldt and Gehring 1998, 2001; Terno et al. 2000; Mack, Bortfeldt, and Gehring 2004; Moura and Oliveira 2005). Moreover, issues related to cargo fragility, in terms of constraints on the load bearing ability of items, have been taken into account by some authors (Ratcliff and Bischoff 1998, Bischoff 2006). Despite the considerable progress that has been made towards meeting the needs of practitioners, however, much work remains to be done before it can be claimed that the approaches suggested in the literature address adequately and fully the range of different problem scenarios which arise in practice. Combining load plan construction heuristics with agent-based simulation approaches is put forward here as a possible contribution towards achieving this aim.

\section{AGENT-BASED SIMULATION}

ABS is a simulation technique that models the overall behavior of a system through use of autonomous system components (also referred to as agents) that communicate with each other by using messages. The behavior incorporated into an agent determines its role in the environment, its interaction with other 


\section{Mustafee and Bischoff}

agents, its response to messages from other agents, and indeed whether its own behavior is adaptable. The agents usually have some properties and, unlike agent behavior - which may or may not be programmed to adapt itself - the properties usually change during the course of the simulation. These agent properties usually change as a result of certain trigger points in the simulation, for example, time triggered changes, changes triggered by an agent's internal state change, changes triggered due to messages being received from other agents, etc. (also applicable to agent behaviors - if they are programmed to change). Since there are usually a number of self-governing agents in an environment, each with the aforementioned characteristics, the overall system state is determined by their dynamic interactions through time. In ABS, the simulation time increments in discrete time steps.

The characteristics of agents and ABS are quite widely discussed in the literature. Macal and North (2006), for instance, consider agents to have six specific characteristics, namely, attributes, behavioral rules, memory, resources, decision making sophistication and rules to modify behavioral rules. The agent behavior, which is defined by simple rules, may be influenced by their interaction with other agents; and this "agent-by-agent and interaction-by-interaction" approach to modeling usually gives rise to emergent "patterns, structures and behaviors" that are not explicitly modeled in the system (Macal and North 2010).

ABS has been applied in several areas. In healthcare, ABS has been used for modeling the spread of pathogens that are transmitted by direct contact (Hotchkiss et al. 2005); Stainsby, Taboada, and Luque (2009) have used agents to model hospital emergency departments. In financial trading, ABS has been used to model new electricity trading arrangements in the UK (Bunn and Oliveira 2001). Agent-Based Social Simulation (ABSS) is the application of ABS in the social sciences context, for example, it has been used for modeling social interactions and influence (Marsella, Pynadath, and Read 2004). Luo et al. (2008) and Moulin et al. (2003) used ABS for crowd management. Further applications of this technique have been reported in supply chain management (Kaihara 2003), in the military (Cioppa, Lucas and Sanchez 2004), for emergency evacuations (Pan et al. 2007), and in cell biology (Pogson et al. 2006), among others.

ABS has also been applied to container management, as opposed to container loading. Henesey (2006) investigated the use of agent-based technologies to improve the performance of container terminals. The use of an ABS architecture to solve the automatic container allocation problem in a port container terminal is described by Rebollo et al. (2000). The use of agents to simulate and optimize cargo handling storage space in a maritime port is reported by Kefi et al. (2009). ABS has been used to model the management of stakeholder relations in container terminals through use of agents that simulate different stakeholder behavior (Henesey, Notteboom, and Davidsson 2003). Henesey, Davidsson and Persson (2006) report on the use of the SimPort ABS tool to evaluate eight transshipment policies. Bin, WenFeng, and Yu (2009) have used ABS, utilizing 14 kinds of agents, to model a container terminal logistics system. The project Container World has modeled both business and operational aspects of the container business in the UK though a multi-agent methodology (Sinha-Ray et al. 2003).

Whilst studies have been conducted in the general area of port and container terminal management, there is, to the best of the authors' knowledge, no previous work using ABS in the specific area of container loading. Thus, the authors are arguably among the first to report on the application of ABS to simulate proximity-based interactions among different items of cargo, such as those resulting from the spatiotemporal changes that occur during the transportation of freight containers.

\section{COMBINING CLA AND ABS: A MULTI-METHODOLOGY APPROACH}

The purpose of the combined CLA and ABS approach to container loading is to find the trade-off between container loading efficiency and various important practical considerations in relation to the cargo such as, its stability, fragility, volatility of cargo, and cross-contamination. As highlighted earlier, there is a need for more research in relation to addressing the different "practical" issues which arise in connection with container loading plans. In this paper, only the knapsack version of the problem is considered, wherein the space available is fixed and loading all the cargo may not be possible. The cargo may consist of items of various degrees of heterogeneity. In the terminology of Wäscher, Haußner, and Schumann 


\section{Mustafee and Bischoff}

(2007), therefore, the problems considered are of type Single Large Object Placement Problem (SLOPP) and Single Knapsack Problem (SKP).

We argue that the individual items of cargo can be modeled as agents because they display three important characteristics consistent with most agents - (1) they have properties, (2) they exhibit autonomous behavior (since each item of cargo is bestowed with the independent Newtonian motion properties), and (3) they interact with other agents and this leads to more complex behavior (interaction is usually brought about by the spatiotemporal changes during transportation). In order to better describe the application context and the structure of our multi-methodology approach to container loading, we consider two issues: (a) the stability of the load as it is being transported, and (b) the possible cross-contamination between different types of items in the cargo over time. With regard to the second issue, we consider a situation where the items to be loaded have differing degrees of perishability and where badly deteriorated items can affect those in their immediate vicinity (e.g., through the spread of mold). Our argument for using ABS to model the two aspects (henceforth referred as cargo stability and cargo cross-contamination scenario, respectively) is summarized in Table 1. It provides details of the three agent characteristics mentioned above for the cargo stability and cross-contamination scenarios.

Table 1: Agent characteristics for the cargo stability and the cargo cross-contamination scenarios

\begin{tabular}{|l|l|l|}
\hline Agent Characteristics & \multicolumn{1}{|c|}{ Cargo Stability Scenario } & Cargo Cross-Contamination Scenario \\
\hline $\begin{array}{l}\text { (1) Agents have prop- } \\
\text { erties }\end{array}$ & $\begin{array}{l}\text { Dimension of the individual items } \\
\text { of cargo (length, breadth, height). }\end{array}$ & $\begin{array}{l}\text { Freshness index (i.e., the length of time } \\
\text { after which the individual items will } \\
\text { start developing mold). }\end{array}$ \\
\hline $\begin{array}{l}\text { (2) Agents exhibit au- } \\
\text { tonomous behavior }\end{array}$ & $\begin{array}{l}\text { The stability of an item is usually } \\
\text { dependent on the dimensions of the } \\
\text { item and other factors (e.g., } \\
\text { weight). }\end{array}$ & $\begin{array}{l}\text { The freshness index will usually de- } \\
\text { crease over time. }\end{array}$ \\
\hline $\begin{array}{l}\text { (3) Interaction between } \\
\text { agents may result in } \\
\text { more complex behavior }\end{array}$ & $\begin{array}{l}\text { The stability of an individual item } \\
\text { may also depend on the stability of } \\
\text { the items that surround/support the } \\
\text { item in question. }\end{array}$ & $\begin{array}{l}\text { Perishable items may develop mold } \\
\text { when they come in contact with other } \\
\text { mold-affected items. }\end{array}$ \\
\hline
\end{tabular}

Having presented the rationale for using ABS to model the loaded container in our example scenarios, we now discuss the overall approach in more detail. It uses two techniques - algorithms for Cutting and Packing Optimization (more specifically, CLAs) and Simulation (i.e., ABS). The methodology is applied in two stages and there are several iterations (see Figure 1). In Phase One, a container loading plan is generated. This should ideally contain the $\mathrm{x}, \mathrm{x}^{\prime}(\mathrm{x}+$ length of box $), \mathrm{y}, \mathrm{y}^{\prime}(\mathrm{y}+$ width of box $), \mathrm{z}$ and z' $(\mathrm{z}+$ height of box) co-ordinates of each item loaded (note that in our knapsack version of the problem not all items may be selected) as well as a corresponding freshness index. Associated with the loading plan is a certain container utilization percentage. This, of course, is a function of the items selected by the CLA. Different CLAs - or separate runs of a single CLA where this involves a random element or allows for different parameter settings - will generally lead to plans with different utilization figures. It is worth noting that the proposed methodology has no underlying dependency on any particular set of CLAs. The only requirement is that the CLA(s) being used in Phase One provide output relating to the physical arrangement of the cargo items in the $\mathrm{x}, \mathrm{y}$ and $\mathrm{z}$ axes (the container loading plan).

In the second stage, the container loading plan generated is used as an input to an ABS model (see Figure 1). Our approach stipulates that the ABS model should have the following functionality: (1) agents to be created for each item listed in the loading plan; (2) agents to be bestowed the mandatory dimension properties - length, width, and height (these can be calculated from the data in the container loading plan) - and one or more optional properties that may be of relevance (e.g., a freshness index); (3) an agent's proximity-interactions with other agents, to be determined on the basis of the agents' $\mathrm{x}, \mathrm{y}$ and $\mathrm{z}$ co-ordinates. Thus, the logic incorporated in the ABS model should be able to determine if, for example, 


\section{Mustafee and Bischoff}

agents representing two items of cargo are in contact with each other in any direction. It may be noted that the agents' dimension properties may also be used for 3D representation of the items of cargo (see section 5 for our implementation); however, visualization is not a requirement of our approach. Another point to note is that our approach has no underlying dependency on any specific ABS software. The only requirement is that the ABS model must be able to support the three functionalities mentioned above.

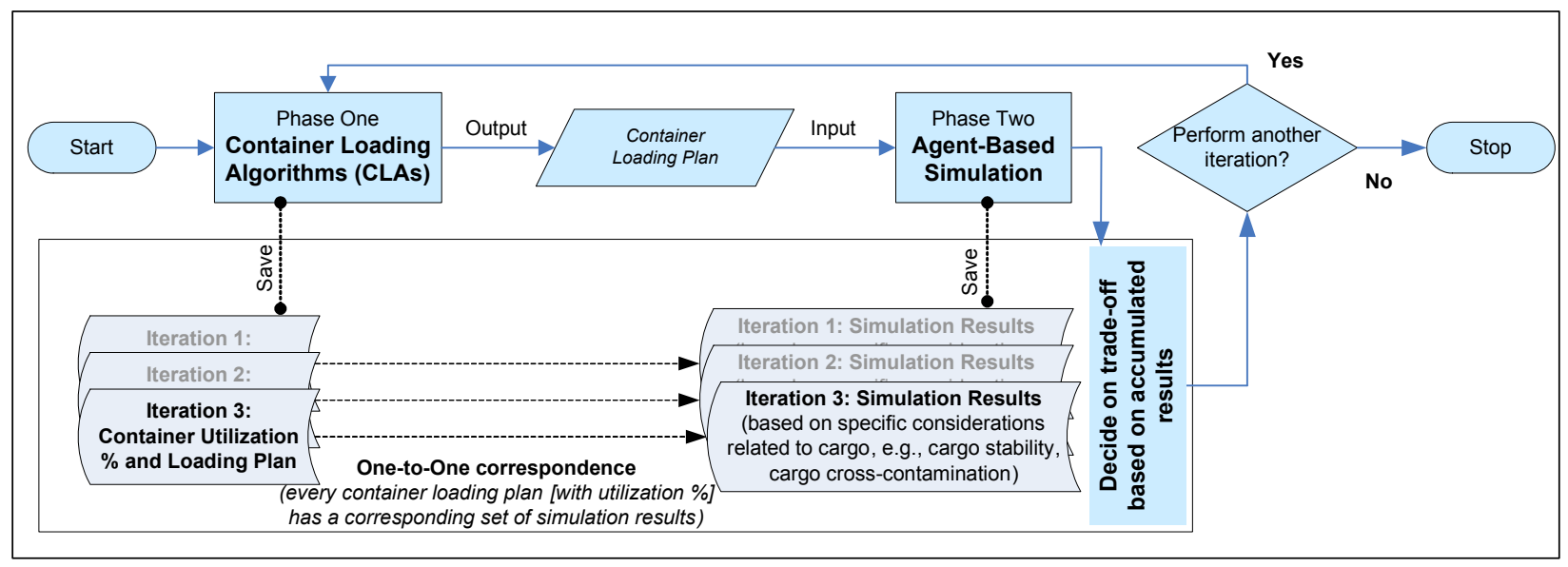

Figure 1: Flowchart of the Multi-Methodology Agent-Based Approach to Container Loading

The agent-based model can be executed to simulate the transport of cargo through time. The results to be collected will depend on the scenario being investigated. With regard to the two example scenarios introduced here, result collection for cargo stability may involve the number of boxes that have dropped off the stack during transportation (i.e., by the end of the simulation run) and in the cross-contamination scenario may involve the number of mold-affected items. Collection of results completes Phase Two of the approach. At this point we have a container utilization percentage (output of CLA(s)) and a related set of results from the ABS (Figure 1). Again referring back to our example scenarios, a utilization of $90 \%$ may have resulted in, say, 25 boxes having fallen down in transit and 45 boxes being affected by mold.

As has already been explained, the purpose of our approach is to determine the trade-off between container loading efficiency and other relevant factors, such as the stability of the load or the likelihood of cross-contamination. Since every execution of the CLA(s) in Phase One will usually result in a different loading plan (and a corresponding utilization percentage) and this plan can be used for the ABS in Phase Two, we can, by conducting several of these Phase One - Phase Two iterations, generate a large amount of data in relation to trade-offs between container loading efficiency and the other considerations. Staying with our cargo stability example, if container utilizations of $67 \%, 78 \%$ and $90 \%$ in Phase One result in 30 , 18 and 25 boxes, respectively, dropping off the stack, it may worthwhile to sacrifice a degree of loading efficiency and use the arrangement associated with a $78 \%$ utilization. Similarly, in the crosscontamination scenario the stakeholders are in a position to make an informed decision about the acceptable trade-off between space utilization and the percentage of spoilt goods. Finally, as with most simulations, the larger the number of Phase One - Phase Two iterations (we do not use the term experiments here since each iteration may involve several ABS experiments/trials when the model is stochastic), the greater the amount of useful data that is available to the decision-maker.

It should be emphasized that the approach does not in itself seek to define the most appropriate tradeoff between the factors involved. This decision is left to the user. However, the approach enables dominated solutions encountered in the course of the computations to be eliminated from the decision-process, leaving the user to choose between 'sensible' alternatives on the calculated approximation to the efficient frontier. Where a user is able to specify a-priori weighting factors for the different criteria considered, 


\section{Mustafee and Bischoff}

these can, of course, be easily incorporated into the approach. In this case the best of the calculated loading plans can be identified automatically without any further user involvement.

\section{THE SIMULATION MODEL}

This section of the paper describes the two ABS models that were used to experiment with Phase Two of the approach. The cargo stability example represents a rudimentary proof-of-concept that uses an opensource physics engine to model the individual items of cargo. For the cross-contamination example a 3-D ABS model has been developed using AnyLogic software (XJ Technologies 2010).

\subsection{Cargo Stability Analysis Using a Physics Engine}

In order to simulate effectively the stability of cargo during transportation, the individual items should be modeled such that they adhere to Newton's laws of motion (Cutnell and Johnson 2001). In such a model the movement of individual items of cargo will depend on their properties, e.g., length, breadth, height, mass/weight, contact friction, etc.. This can be modeled through the use of a physics engine like Jinngine (Silcowitz-Hansen 2010). A further aspect of modeling cargo stability adequately concerns the effects of collisions between objects. Some physics engines like Jinngine also provide collision detection support.

A stack of boxes arranged inside a container (Figure 2 - left: a pallet is used here to illustrate the effects more clearly) can be simulated through time if: (a) every box is transformed into an agent - this may require the use of ABS software, (b) every box is bestowed the independent Newtonian motion properties (its motion will, in turn, depend upon the agents' properties, e.g., dimensions, weight, etc.) - this may be accomplished through the use of a physics engine - and (c) the proximity-based interactions among the boxes is captured using collision detection techniques - this can be accomplished through use of software that supports collision detection. The resultant simulation could potentially determine the degree of cargo stability based on the number of boxes that may have fallen off the stack during transportation (when visually represented in 3D, this may look like Figure 2 - right).
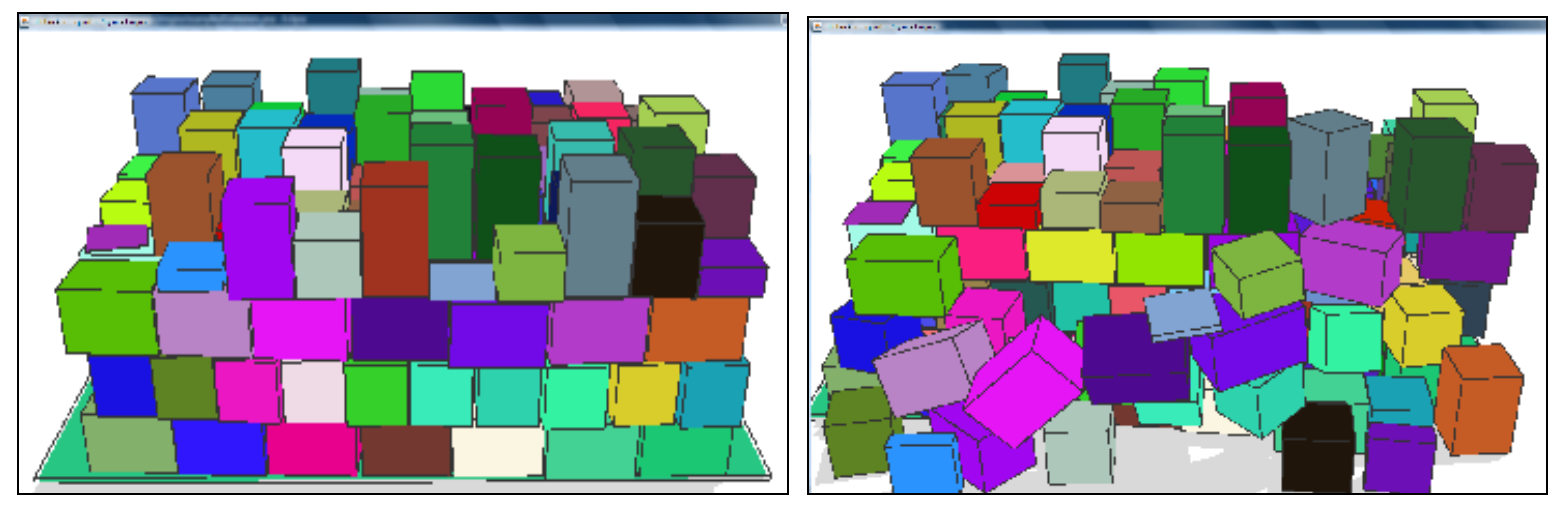

Figure 2: The stack of boxes while at rest (left) and subsequent to external stimulus being applied (right)

It must be pointed out that the proof-of-concept program that was used to generate the screenshots above uses Jinngine for (b) and (c); it does not include any ABS agent functionality. We are, therefore, unable to simulate the model through time. The screenshot presented in Figure 2 (right) is the result of an external stimulus (pull) being applied to the base of the pallet in an instant in time. Thus, the example is more akin to a 3-D game that incorporates objects and collision detection. It is not a true ABS simulation.

Finally, although the boxes in our example program are not modeled as ABS agents, they do however display the important characteristics that are found in most agents, namely: (a) they have properties (the boxes are of different dimensions and colors), (b) they display autonomous behavior (the boxes display independent Newtonian motion, which is, in turn, dependent on their properties), and (c) inter-agent interaction results in a more complex behavior (the collision among boxes will result in some boxes toppling 


\section{Mustafee and Bischoff}

over, which may, in turn, affect the other boxes in the load). Future work could involve the integration of ABS software with Jinngine in order to perform rigid body simulation of items through time.

\subsection{Modeling Cargo Cross-Contamination Using AnyLogic}

The AnyLogic model developed by the authors is informed by the functionality proposed by the multimethodology approach for Phase Two ABS (refer to section 4). Namely, (1) the model reads the Phase One loading plan generated by the CLA(s) and creates a unique agent for each item of cargo listed in the plan; (2) each agent is provided with four properties - length, breadth, height and a freshness index (the items of cargo/agents and the surrounding containers are represented visually in 3 dimensions); (3) for each agent created, the ABS model identifies all the other agents surrounding the agent in question and establishes a relationship between them (this inter-agent relationship is used during the simulation to pass messages among the agents). Figure 3 (left) shows a randomly-selected agent (red box), and eight other agents surrounding this agent; these proximity relationships are identified by the model on the basis of the $\mathrm{x}, \mathrm{x}^{\prime}, \mathrm{y}, \mathrm{y}^{\prime}, \mathrm{z}$ and z' coordinates of the different items, and these inter-agent relationships are subsequently stored in the model. The model is run for 30 days in simulation time.

Every agent has a freshness index that was allocated in Phase One. This index is a randomly generated value between 5 and 60 (generated from a discrete uniform distribution; this is for illustration purposes only) and it is assigned to different box types, i.e., all boxes of the same type have the same freshness index. The index is used to denote the number of simulated days for which the contents of the box will remain mold free. Thus, as the simulation progresses in time, at the end of every simulated day, the freshness value associated with every agent is decreased by one (if freshness $>0$ ). When the freshness level of a particular box reaches zero, it is said to be mold affected. The color of the box is changed in the 3-D space so as to enable easier identification (Figure 3 - right).
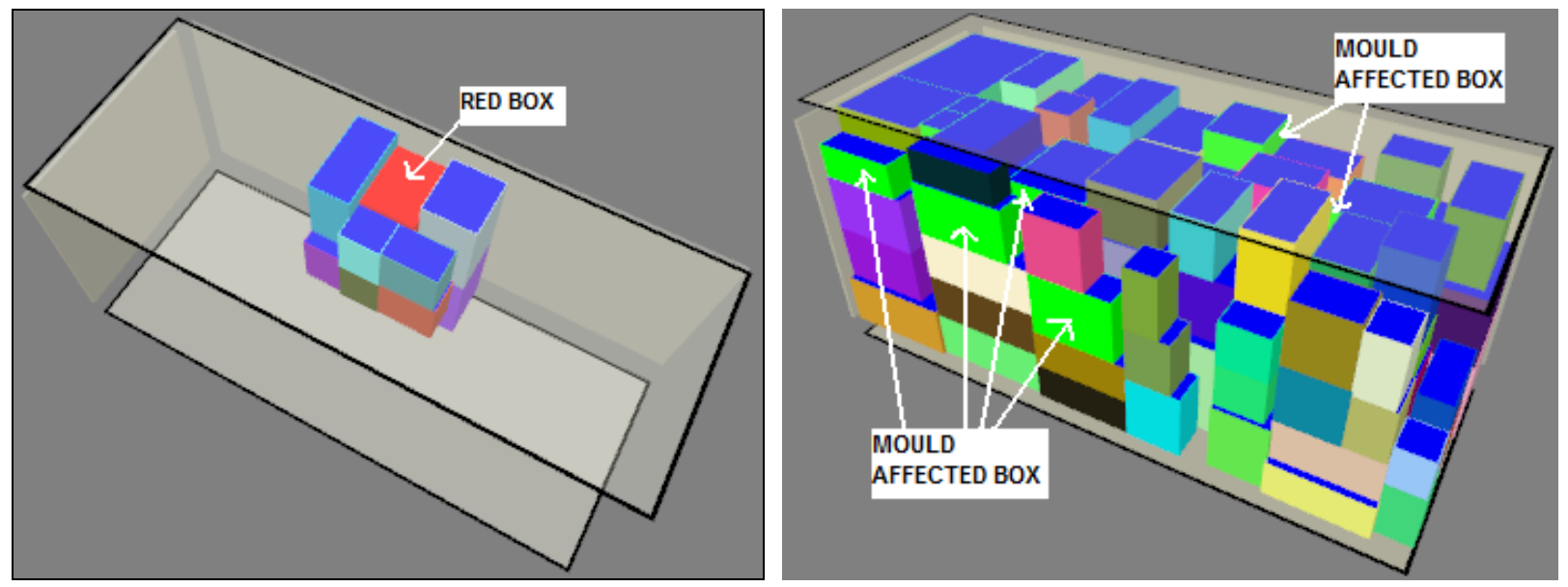

Figure 3: Detection of proximity among agents (left); Mold-affected boxes during simulation (right)

As soon as a box becomes affected by mold it starts to contaminate other boxes which physically surround it. This contamination is reflected in the model by the proximity-based interactions sent by the agent to all the surrounding agents. Thus, in Figure 3 (left), if the red box/agent were to become moldaffected, it will immediately send messages to all the eight boxes/agents surrounding it. The message is a signal to the other agents (each of whom have their own copy of freshness index that decreases over time) that they have now been contaminated. Upon receiving this message, each agents immediately decreases the existing value of its freshness index by 1 (if freshness index $>0$ ). This is in addition to the drop of 1 unit of freshness that is applied at the end of each simulated day. The logic of the model, as described above, is repeated until the end of the simulation period. Several pieces of data are then collected, including the number of mold-affected boxes (Figure 4 - left), the maximum freshness value associated with an 


\section{Mustafee and Bischoff}

item of cargo, average cargo freshness, etc.. A histogram of the freshness data can be produced easily (Figure 4 - right). This completes a single ABS Phase two iteration. As has been described earlier, our multi-methodology approach suggests that several such iterations be performed, each with a different container loading plan, so as to enable the stakeholder to decide on a trade-off between the container efficiency and other considerations related to cargo. Experiments and results are described next.

\section{EXPERIMENTS AND RESULTS}

This section describes the application of the proposed approach to the cargo cross-contamination scenario. Phase One of our methodology employs the CLA originally proposed by (Bischoff 2006). It involves a random search process and as such is capable of producing a large number of different loading plans (with differing degrees of container utilization). Due to space restrictions no details of the algorithm itself are presented here. Both the goods and the container are assumed to be rectangular in shape and have known dimensions. The algorithm makes no intrinsic assumptions about the degree of heterogeneity in the cargo, but was put forward in the context of weakly heterogeneous mixes. The items ("boxes") are assumed to belong to different "box types". The predominant factor that distinguishes the various box types are the boxes' physical dimensions, i.e., length, breadth and height, and its load bearing strength. For the purpose of the cargo cross-contamination scenario presented in this paper, the load bearing strength is ignored and a new property - the freshness index (section 4) - is introduced. This is used only in Phase Two of our methodology. The associated ABS model has already been described in section 5.2. The reader is reminded that several Phase One - Phase Two iterations may be required to enable the stakeholder to take an informed decision regarding the trade-off between efficient cargo utilization and the post-transit degree of contamination (refer to Figure 1). In our experiments the number of such iterations were limited to five.

For our experiments we have used a total of four benchmark problems, labeled BTM20, BTM40, BTM70 and BTM100. Each represents a specific Box Type Mix (BTM) with 20, 40, 70 and 100 box types respectively. Using the CLA referred to above we generated 100 container loading plans for each of our four BTMs. We then ranked the container utilization rates for these loading plans, and selected five specific ones for every BTM. These are as follows (where, $x=20,40,70,100$ ): (a) plan with the lowest container utilization (BTMxmin); (b) plan with the highest utilization (BTMxmax); (c) plan with utilization percentile rank of 25 (BTMx25); (d) plan with utilization percentile rank of 50 (BTMx50); and (e) plan with utilization percentile rank of 75 (BTM $x 75)$. Each BTM represents a unique experiment group; each group comprises of five separate iterations, wherein each Phase Two iteration uses a unique container loading plan. The iterations will henceforth be identified by the BTM number and a corresponding loading plan. For example, BTM40max will refer to the iteration using the container loading plan with the highest container utilization ( $\max$ ) for experiment group BTM40.

Figure 4 (left) shows the percentage of mould-affected items for the four experiments (BTMs). Although the results for all the BTMs are presented in the same figure, comparisons cannot be made across BTMs since the freshness indices of the cargo items change from one experiment type to the next. The graph shows the existence of trade-offs between container loading efficiency and the percentage of boxes affected with mold. For example, in relation to the BTM20 experiments, the five loading plans selected for simulation had utilization efficiencies of $58.79 \%, 78.93 \%, 82.60 \%, 84.43 \%$ and $89.94 \%$, respectively; the corresponding proportions of mold-affected boxes were $65.15 \%, 72.22 \%, 70.65 \%, 69.79 \%$ and $76 \%$ (shown in Figure 4 - left). Thus, changes in container utilization rates (brought about by a change in the loading plan) do have an effect on the percentage of items that are spoilt. Our approach has the potential to help the stakeholder to take an informed decision on the loading plan to use, as it provides the container utilization and the corresponding simulation data from multiple iterations. Let us consider the trade-offs for our BTM20 experiments. The stakeholder, having seen the results of five iterations, may decide to use the loading plan that provides $84.43 \%$ utilization efficiency (BTM2075) - and not BTM20min which provides $58.79 \%$ efficiency but has the lowest percentage of boxes affected with mold. This may be because with the BTM2075 loading plan the stakeholder will be able to transport more boxes (96 as against 66 if 


\section{Mustafee and Bischoff}

he chose BTM20min), and this may make up for the slight increase in the possible degree of contamination for the chosen scheme. The results for all the four BTMs also demonstrate the absence of any clear (positive or negative) correlation between container utilization and the percentage of boxes affected with mold.
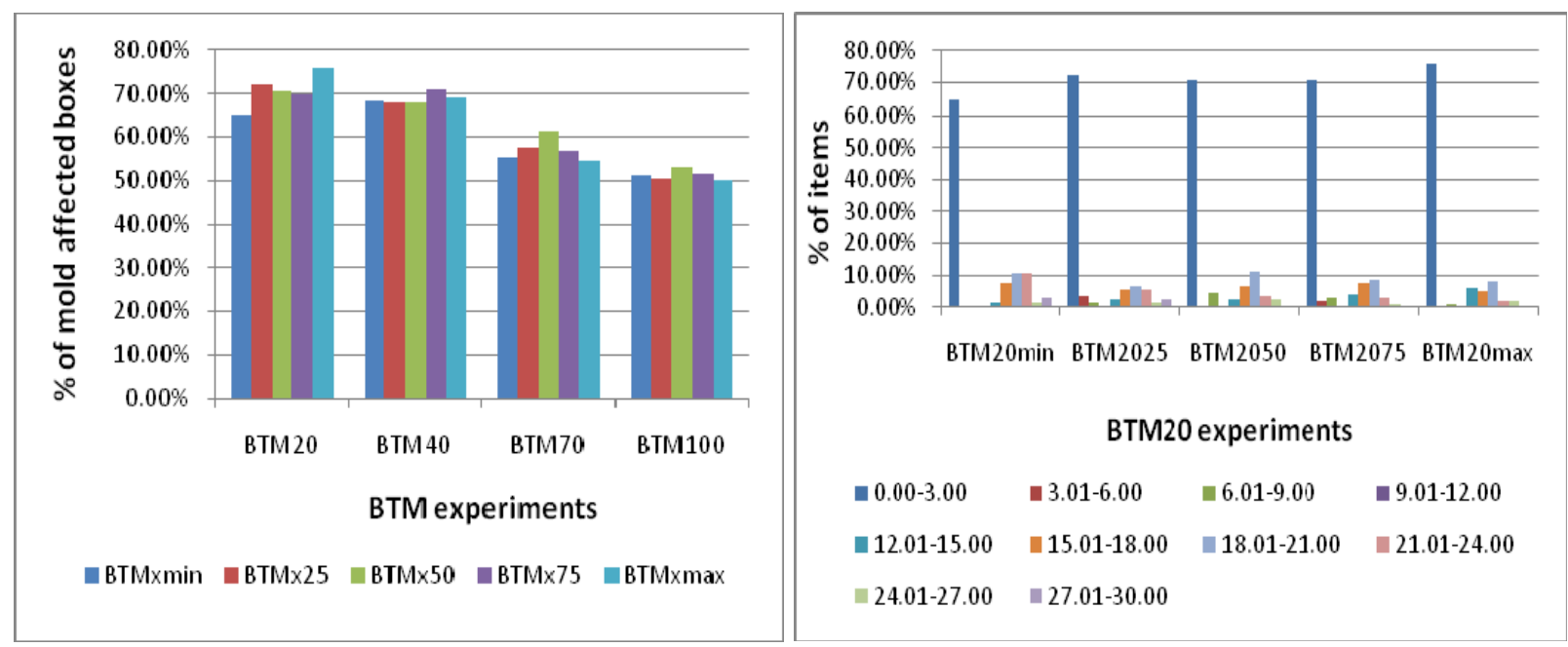

Figure 4: \% mold-affected boxes, grouped by BTM (left); Histogram showing freshness \% for BTM20 at the end of simulation (right)

Figure 4 (right) presents a histogram of freshness values for the BTM20 experiment. It shows that for all the five loading plans simulated, between 65 to $76 \%$ of items had already developed mold or would be mold-affected in the next three days (note the freshness/bin range: 0.00-3.00). It further shows that if the BTM20min loading plan was selected, then approximately $33.33 \%$ of items will remain relatively fresh (bin/freshness range: 15.00 to 30.00 ) by the end of the 30 -day simulation - and this value is at least $50 \%$ higher when compared to the corresponding freshness values of the alternate loading plans. Here again there may be an opportunity for a trade-off and the decision of the stakeholder may be based on practical business considerations.

\section{CONCLUSION}

This paper has presented a multi-methodology agent-based approach to container loading. It involves combining container loading algorithms with agent-based simulation. The purpose of this approach is to enable the stakeholders to analyze the trade-offs between loading efficiency and other cargo-related considerations. A feasibility study was conducted in order to test the approach. It investigated the trade-off between container utilization and the cross-contamination among boxes containing perishable goods. The multi-methodology approach is realized through use of a container loading algorithm from the literature and the development of an ABS model in AnyLogic that simulates cargo cross-contamination by modeling the propagation of mould inside the container. The results of the experiments demonstrated the tradeoffs involved in container utilization and the number of mold-affected boxes. The results also showed that there was no correlation between these variables.

The contribution of this research is the novel application of the multi-methodology agent-based approach to container loading (section 4). The purpose of the combined CLA and ABS approach is to find the trade-off between container loading efficiency and various important practical considerations in relation to the cargo - such as, its stability, fragility, volatility of cargo, and cross-contamination. Further contributions of this research are the feasibility study on cargo efficiency and cross-contamination (sections 5.2 and 6). 


\section{Mustafee and Bischoff}

Finally, we draw attention to future work which could be derived from what has been presented in this paper. Here we have used the CLAs and the cross-contamination example to demonstrate our proximity-based ABS modeling approach. However, this approach can be expanded to model analogous situations wherein physical proximity between agents is the key condition for inter-agent interaction - for example, spread of fire through hazardous materials. Moreover, this paper has identified and discussed the components required to facilitate simulation of container stability, namely, ABS software, Physics engine and collision detection software (section 5.1). Future work could involve the integration of ABS software with tools such as the Jinngine Physics engine in order to perform rigid body simulation of items through time.

\section{REFERENCES}

Beasley, J. $\quad$ E. $1996 . \quad$ "OR-Notes." Accessed March 22. http://people.brunel.ac.uk/ mastjjb/jeb/or/contents.html.

Bin, L., L. Wen-Feng, and Z. Yu. 2009. "Study on Modeling of Container Terminal Logistics System Using Agent-Based Computing and Knowledge Discovery." International Journal of Distributed Sensor Networks 5(1):36-36.

Bischoff, E. E. 2006. "3-D Packing of Items with Limited Load Bearing Strength." European Journal of Operational Research 168(3):952-966.

Bischoff, E. E., and M. S. W. Ratcliff. 1995. "Issues in the Development of Approaches to Container Loading." Omega 23(4):377-390.

Bortfeldt, A., and H. Gehring. 1998. "Ein Tabu Search-Verfahren für Containerbelade-probleme mit schwach heterogenem Kistenvorrat." Operations Research Spektrum 20(1998):237-250.

Bortfeldt, A., and H. Gehring. 2001. "A Hybrid Genetic Algorithm for the Container Loading Problem." European Journal of Operational Research 131(1):143-161.

Bunn, D. W., and F. S. Oliveira. 2001. "Agent-Based Simulation-An Application to the New Electricity Trading." IEEE Transactions on Evolutionary Computation 5(5):493-503.

Cioppa, T. M., T. W. Lucas, and S. M. Sanchez. 2004. "Military Applications of Agent-Based Simulations." In Proceedings of the 2004 Winter Simulation Conference, edited by R .G. Ingalls, M. D. Rossetti, J. S. Smith, and B. A. Peters, 171-180. Piscataway, New Jersey: Institute of Electrical and Electronics Engineers, Inc.

Cutnell, J. D., and K. W. Johnson. 2001. "Forces and Newton's Laws of Motion." Chap. 4 in Physics. New York: John Wiley \& Sons.

Davies, A. P., and E. E. Bischoff. 1999. "Weight Distribution Considerations in Container Loading." European Journal of Operational Research 114(3):509-527.

Egeblad, J., C. Garavelli, S. Lisi, and D. Pisinger. 2010. "Heuristics for Container Loading of Furniture." European Journal of Operational Research 200(3):881-892.

Eley, M. 2002. "Solving Container Loading Problems by Block Arrangement." European Journal of Operational Research 141(2):393-409.

Fanslau, T., and A. Bortfeldt. 2010. "A Tree Search Algorithm for Solving the Container Loading Problem." INFORMS Journal on Computing 22(2):222-235.

Gehring, H., and A. Bortfeldt. 1997. "A Genetic Algorithm for Solving the Container Loading Problem." International Transactions in Operational Research 4(5/6):401-418.

Gehring, H., and A. Bortfeldt. 2002. "A Parallel Genetic Algorithm for Solving the Container Loading Problem." International Transactions in Operational Research 9(4):497-511.

Gehring, H., K. Menschner, and M. Meyer. 1990. "A Computer-Based Heuristic for Packing Pooled Shipment Containers." European Journal of Operational Research 44(2):277-288.

George, J. A. 1992. "A Method for Solving Container Packing for a Single Size Box." Journal of the Operational Research Society 43(4):307-312. 
George, J. A., and D. F. Robinson. 1980. "A Heuristic for Packing Boxes into a Container." Computers \& Operations Research 7(3):147-156.

Han, C. P., K. Knott, and P. J. Egbelu. 1989. "A Heuristic Approach to the Three-Dimensional CargoLoading Problem." International Journal of Production Research 27(5):757-774.

Henesey, L. E. 2006. "Multi-Agent Systems for Container Terminal Management." PhD Thesis, Department of Systems and Software Engineering, School of Engineering, Blekinge Institute of Technology. Blekinge Institute of Technology Doctoral Dissertation Series No 2006:08.

Henesey, L. E., P. Davidsson, and J. A. Persson. 2006. "Agent Based Simulation Architecture for Evaluating Operational Policies in Transshipping Containers." Lecture Notes in Computer Science 4196/2006 (Multiagent System Technologies), edited by K. Fischer, I. Timm, E. André, and Z. Ning. Heidelberg, Berlin: Springer.

Henesey, L. E., T. E. Notteboom, and P. Davidsson. 2003. "Agent-Based Simulation of Stakeholders Relations: An Approach to Sustainable Port Terminal Management." In Proceedings of the 2003 International Association of Maritime Economists Annual Conference, 314-331.

Hotchkiss, J. R., D. G. Strike, D. A. Simonson, A. F. Broccard, and P. S. Crooke. 2005. "An Agent-Based and Spatially Explicit Model of Pathogen Dissemination in the Intensive Care Unit." Critical Care Medicine 33(1):168-176.

Huang, W., and K. He. 2009. "A Caving Degree Approach for the Single Container Loading Problem." European Journal of Operational Research 196(1):93-101.

Kaihara, T. 2003. "Multi-Agent Based Supply Chain Modelling with Dynamic Environment." International Journal of Production Economics 85(2):263-269.

Kefi, M., K. Ghedira, O. Korbaa, and P. Yim. 2009. "Container Handling Using Multi-Agent Architecture." International Journal of Intelligent Information and Database System 3(3):338-360.

Luo, L., S. Zhou, W. Cai, M. Y. H. Low, F. Tian, Y. Wang, X. Xiao, and D. Chen. 2008. "Agent-Based Human Behavior Modeling for Crowd Simulation." Computer Animation and Virtual Worlds 19(34):271-281.

Macal C. M., and M. J. North. 2006. "Tutorial on Agent-Based Modeling and Simulation Part 2: How to Model with Agents." In Proceedings of the 2006 Winter Simulation Conference, edited by L. F. Perrone, F. P. Wieland, J. Liu, B. G. Lawson, D. M. Nicol, and R. M. Fujimoto, 73-83. Piscataway, New Jersey: Institute of Electrical and Electronics Engineers, Inc.

Macal C. M., and M. J. North. 2010. "Tutorial on Agent-Based Modelling and Simulation." Journal of Simulation 4(3):151-162.

Mack, D., A. Bortfeldt, and H. Gehring. 2004. "A Parallel Hybrid Local Search Algorithm for the Container Loading Problem." International Transactions in Operational Research 11(5):511-533.

Marsella, S.C., D. V. Pynadath, and S. J. Read. 2004. "PsychSim: Agent-Based Modeling of Social Interactions and Influence." In Proceedings of the International Conference on Cognitive Modeling, 243248. Pittsburgh, PA, USA.

Morabito, R., and M. Arenales. 1994. "An And/Or-Graph Approach to the Container Loading Problem." International Transactions in Operational Research 1(1):59-73.

Moulin, B., W. Chaker, J. Perron, P. Pelletier, J. Hogan, and E. Gbei. 2003. "MAGS Project: Multi-agent GeoSimulation and Crowd Simulation." In Lecture Notes in Computer Science 2825/2003, edited by W. Kuhn, M. Worboys, and S. Timpf. Heidelberg, Berlin: Springer.

Moura, A., and J. F. Oliveira. 2005. "A Grasp Approach to the Container Loading Problem." IEEE Intelligent Systems 20(4):50-57.

Ngoi, B. K. A., M. L. Tay, and E. S. Chua. 1994. "Applying Spatial Representation Techniques to the Container Packing Problem." International Journal of Production Research 32(1):111-123.

Pan, X., C. S. Han, K. Dauber, and K. H. Law. 2007. "A Multi-Agent Based Framework for the Simulation of Human and Social Behaviors During Emergency Evacuations." AI \& Society 22(2):113-132.

Parreño, F., R. Alvarez-Valdes, J. M. Tamarit, and J. F. Oliveira. 2008. "A Maximal-Space Algorithm for the Container Loading Problem.” INFORMS Journal on Computing 20(3):412-422. 
Pisinger, D. 2002. "Heuristics for the Container Loading Problem." European Journal of Operational Research 141 (2):292-382.

Pogson, M., R. Smallwood, E. Qwarnstrom, and M. Holcombe. 2006. "Formal Agent-Based Modelling of Intracellular Chemical Interactions." Biosystems 85(1):37-45.

Ratcliff, M. S. W., and E. E. Bischoff. 1998. "Allowing for Weight Considerations in Container Loading." Operations Research Spektrum 20(1):65-71.

Rebollo, M., V. Julian, C. Carrascosa, and V. Botti. 2000. "A Multi-Agent System for the Automation of a Port Container Terminal." In Proceeding of the Autonomous Agents 2000 Workshop on Agents in Industry. Barcelona.

Silcowitz-Hansen, M. 2010. "Jinngine, a Physics Engine Written In Java." Accessed March 29. http://code.google.com/p/jinngine/.

Sinha-Ray, P., J. Carter, T. Field, J. Marshall, J. Polak, K. Schumacher, D. Song, J. Woods, and J. Zhang. 2003. "Container World: Global agent-based modelling of the container transport business." In Proceedings of the 4th Workshop on Agent-Based Simulation, Edited by M.-M. Seidel. SCS Europe BVBA.

Stainsby, H., M. Taboada, and E. Luque. 2009. "Towards an Agent-Based Simulation of Hospital Emergency Departments." In Proceedings of the 2009 IEEE International Conference on Services Computing, 536 - 539. IEEE.

Terno, J., G. Scheithauer, U. Sommerweiss, and J. Riehme. 2000. "An Efficient Approach for the MultiPallet Loading Problem." European Journal of Operational Research 123(2):372-381.

Wang, Z., K. W. Li, and J. K. Levy. 2008. "A Heuristic for the Container Loading Problem: A TertiaryTree-Based Dynamic Space Decomposition Approach." European Journal of Operational Research 191(1):86-99.

Wascher, G., H. Haußner, and H. Schumann. 2007. "An Improved Typology of Cutting and Packing Problems." European Journal of Operational Research 183(3):1109-1130.

XJ Technologies. 2010. "XJ Technologies Homepage - AnyLogic." Accessed March 27. http://www.xjtek.com/.

\section{AUTHOR BIOGRAPHIES}

NAVONIL MUSTAFEE is a lecturer in Information Systems and Operations Management in the School of Business and Economics, Swansea University (UK). Prior to this he worked as a Research Fellow in the School of Information Systems, Computing and Mathematics in Brunel University. His research interests are in simulation methodologies, healthcare simulation, parallel and distributed simulation, technology adoption and diffusion, and grid computing. His e-mail address is n.mustafee@swansea.ac.uk.

EBERHARD BISCHOFF holds the Chair of Management Science at Swansea University, UK. He has also had positions there as Director of the Business School and the MBA Program and held appointments at a number of other institutions, such as the International Institute for Applied Systems Analysis, Austria, and Stuttgart University, Germany. He has been conducting research on Industrial Cutting and Packing Problems since the early 1980s. His other research interests include Tourism Management and Multiple Criteria Decision-Making. He can be contacted at e.lydiard@swansea.ac.uk. 